\title{
Routledge International Handbook of Consumer Psychology
}

\begin{tabular}{|r|l|}
\hline Journal: & Qualitative Market Research \\
\hline Manuscript ID & QMR-09-2018-0109 \\
\hline Manuscript Type: & Book Review \\
\hline & \\
\end{tabular}

SCHOLARONE ${ }^{\text {m }}$

Manuscripts 


\section{Book review: Routledge International Handbook of Consumer Psychology}

Consumption is a fundamental characteristic of human life. Indeed, all human behaviours are (in)directly linked to consumption (Tatzel, 2003). However, unlike other animals, human consumption is not solely driven by biological necessity. Indeed, the majority of consumer behaviours are driven by complex psychological processes. Understanding these processes is the remit of Consumer Psychology. A recent addition to the scholarly literature in this field is the Routledge International Handbook of Consumer Psychology.

The Routledge International Handbook of Consumer Psychology aims to provide "an outline of the current state of research on a wide range of areas within consumer psychology" (p. xi). Edited by Cathrine V. Jansson-Boyd and Magdalena J. Zawisza, the handbook comprises of 34 chapters and boasts contributions from 71 different authors across 11 different countries. The handbook is divided into seven sections; 1 ) cross cultural research, 2) consumer cognition and perception, 3) social perception, self, identity and well-being, 4) social and cultural influences, 5) decision-making, attitudes and behavioural research, 6) products, branding preferences and sustainability and 7) internet and electronic media.

Section 1, entitled "introduction and cross-cultural research" is composed of three chapters. The opening chapter by Jansson-Boyd and Marlow introduces the topic of consumer psychology and charts the historical rise of consumerism which precipitated its emergence. En route, the authors identify influential thinkers and psychologists who have contributed to the historical development of consumer psychology. Chapters 2 and Chapter 3 build upon the foundational platform of Chapter 1 by assessing how culture acts as a driver of consumption. In Chapter 2, Gupta provides a useful historical review of studies that highlight the cultural implications of consumption. Helpfully, the author uses a table to itemise the main findings of each landmark study. Presented in chronological order, the table allows the reader to quickly appraise the relevant literature and gain an appreciation of the research themes explored thus far. In Chapter 3, Torelli and Rodas extend the discussion on cultural temporally forward to the present day. Here, the authors reflect upon the onerous task of branding to todays, multicultural consumers. This final chapter in the section discusses how globalisation and multi-culturalism have increased both the psychological complexity of consumers and the branding efforts required to target them.

Section 2, entitled "consumer cognition and perception" comprises of five chapters. The first two chapters of the section are devoted to explaining how sensory information influences consumer perception. While Hutchinson and colleagues consider the importance of visual information within consumer environments (Chapter 4), Jansson-Boyd explains how tactile information can influence product evaluations (Chapter 5). In Chapter 6, the conversation turns to consumer cognition and more specifically, mindsets. Here, Wyer outlines how consumer mindsets can unconsciously colour perceptions and influence decision-making. In Chapter 7, Dimofte broadens the discussion on unconscious cognition by reviewing the literature pertaining to unconscious goal activation and unconscious attitude formation. The final chapter of Section 1 (Chapter 8), Maison and Gregg reflect on the utility of the Implicit Association Test (IAT) as a tool for uncovering unconscious bias. This review of the empirical evidence relating to the IAT nicely compliments the theory driven discussions of the two preceding chapters.

Sections 3 entitled "social perception, self, identity and well-being" is an assortment of six chapters that illustrate how consumer identifies are situationally constructed. To kick off proceedings, Belk examines the rise of the digital self within contemporary consumer culture (Chapter 9). The author draws our attention to the symbiotic relationship between the self and 
consumption. This chapter lays the theoretical ground for more technical considerations relating to self-concept and the consumption of brands (e.g. chapter 10 and chapter 12). For example, in Chapter 10, Siry and colleagues revisit self-congruity theory and try to flesh out the reasons behind the mixed results from studies linking self-concept and brand image. In a similar spirit, Zawisza (Chapter 12) propose model extensions to account for the mixed findings within the social perception literature (see Stereotype Content Model (SCM) and the Brands as Intentional Framework (BIAF)). The remaining chapters in this section (chapters 11, 13 and 14) collectively focus on one theme; how media exposure influences consumer welfare. This interesting trilogy of chapters interrogate the problem from different perspectives. For example, in Chapter 11, Häfner demonstrate that consumer's perceptions of beauty are often the result of social comparisons induced by adverts. This theme is further developed by Hurst and colleagues who examine the deleterious effects of media exposure on body image (Chapter 13). The final chapter by SpiteriCornish (Chapter 14) catalogues the psychological processes that regulate healthy eating habits. Importantly, this chapter also discusses potential policies and interventions that may help stem the tide of rising obesity among consumers.

Section 4, entitled "social and cultural influences" considers different socio-cultural phenomena that influence consumer behaviours. In this section, each of the four chapters are thematically distal from its peers. For example, while Cwalina and colleagues (Chapter 15) consider the theoretical and methodological distinctions between political marketing and mainstream marketing, Zaleskiewicz and Gasiorowska review the psychological impact of money on economic and social relationships (Chapter 17). The two remaining chapters in this section make valuable contributions to the handbook. In Chapter 16, Minton and Kahles consider the consumptive implications of religion (Chapter 16) while Mizerski and colleagues reflect on the vulnerability of children consumers in the $21^{\text {st }}$ century. Both chapters make for interesting reading.

Section 5, entitled "decision making, attitudes and behavioural research" is the largest section of the book. Containing eight chapters, this section invokes various (information processing, motivation and emotion) theories in order to explain the consumptive decisions of shoppers. In the opening chapter (Chapter 19), Krüger and colleagues analyse how message-framing effects can influence responses to persuasive communications. Not to be outdone, in Chapter 21, Teeny and colleagues follow up with an equally strong chapter that also has persuasion as its key theme. The authors focus their attention on the Elaboration Likelihood Model (ELM) which has occupied a central position within persuasion literature for almost 40 years. Reviewing the relevant literature, the authors demonstrate that decision making is heavily reliant upon situational affordances. In Chapter 20, the focus turns to self-regulation as Gollwitzer and colleagues consider the power of implementation intensions to ameliorate consumer decision-making.

Other chapters in Section 5 tackle decision-making from alternative perspectives. For example, in Chapter 22, Kamleitner and Ruzeviciute explore transactional trade-offs while in Chapter 23, Deval and colleagues consider the native theories that underpin consumer inferences. Section 5 finishes strongly with three thought provoking chapters that examine the role of self-control (Chapter 24), sex drive (Chapter 25) and age (Chapter 26) in shaping consumer responses. In Chapter 24, Labroo and Pocheptsova reveal the multitude of self-control dilemmas that weaken resistance and compromise resolve. This body of research illustrates the vulnerability of consumers as actors in the marketplace. Our animalistic fallibility is again brought into sharp relief in Chapter 25 when Chen and Lui considers the bounded logic enforced by our sex drive. In the final chapter of the section, Carpenter and Yoon consider the cognitive challenges associated with old age and identify marketing solutions that may help the greying consumer population. 
Section 6 entitled "products, branding preferences and sustainability" contains an eclectic mix of 5 chapters. The first two chapters in this section tackle the topic of sustainability. The opening chapter by Gurel-Atay and colleagues (Chapter 27) discusses how shifting consumer values have prompted a groundswell of support for sustainable consumption among consumers. Continuing with the theme of sustainability, Friman and Gärling examine the public transport sector and assess how (public) transport is operationalised in different countries. The final three chapters of the section abandon the sustainability theme in order to explore various other themes. In Chapter 29, Florack and Palcu consider the psychology behind branding. Here, the authors outline the importance of brands by mapping the functional, symbolic and experiential benefits that brands bestow upon consumers. In Chapter 30, Reimann and Cao review the "antecedents, processes and behavioural consequences" of aesthetics while Aggarwal and McGill (Chapter 31) discuss how anthropomorphism can the shape the perceptions of consumers and society at large.

Section 7 entitled "Internet and electronic media" is the final section of the book. Composed of three chapters this final section considers how digital environments can shape consumption patterns. In Chapter 32, Reczek and Lamberton consider the social influences embedded within digital environments and the costs/benefits of sharing online content. In a similar vein, Stotler and Dill-Shackleford (Chapter 33) investigate how representations of race, gender and sexual orientation are used in online gaming. In the final chapter of the book (Chapter 34), Matz discusses the growth of personality-customized advertising in the digital environments. This chapter uses cases studies to demonstrate the use of online targeting is particularity interesting in light of the recent controversies surrounding data protection and online privacy.

Overall, it is hard not to be impressed by the Routledge International Handbook of Consumer Psychology. The handbook has sufficient scope and depth to capture the diversity and richness that lies within the field of Consumer Psychology. The publication of this handbook is a welcome and timely addition to the scholarly literature. It has been 10 long years since the Handbook of Consumer Psychology (editors: Cuurtis P. Haugtvedt, Paul M. Herr and Frank Kardes) first provided scholars with an authoritative overview of developments within the field of Consumer Psychology. Much has changed in the intervening years but the Routledge International Handbook of Consumer Psychology bridges the gap with grace. It is a worthy successor to that original text. Importantly, the handbook does not simply provide us with a much needed view of the current state of research. It also casts an eye to the future. Laudably, the majority of contributors take the time to identify gaps in the current literature and signpost future research avenues in the closing paragraphs. Such information is likely to be of particular interest to early stage researchers. In sum, the Routledge International Handbook of Consumer Psychology book is an invaluable resource for marketing students and consumer psychologists alike. The book should be a first port of call for those who wish to get to grips with this fascinating research area. 